\title{
Due Curve di Potenza \\ Rappresentanti l'Energia \\ di un Ciclo di Vita \\ nella Teoria Evo-SETI
}

Claudio Maccone

International Academy of Astronautics (IAA, Parigi)

e Istituto Nazionale di Astrofisica (INAF, Italia)

Correspondence | claudio.maccone@gmail.com

Citation | Maccone, Claudio. 2020. "Due Curve di Potenza Rappresentanti l'Energia di un Ciclo di Vita nella Teoria Evo-SETI” Journal of Big History IV (2): 106-128.

DOI | https://doi.org/10.22339/jbh.v4i2.4251

\section{Riassunto}

Questo articolo presenta un nuovo modello matematico per l'energia di cui un essere vivente ha bisogno per vivere tutto il suo ciclo di vita, tra la nascita e la morte. Questo modello funziona anche con una civiltà fatta di molti esseri viventi. Il modello si basa su una CURVA DI POTENZA LOGELL, cioè una curva nel tempo composta da una densità di probabilità lognormale tra la nascita e il picco, seguita da un'ellisse tra il picco e la morte (LOGELL significa LOGnormale più ELLisse). Noi deriveremo le equazioni analitiche rappresentanti l'energia in termini di tre soli parametri: l'istante della nascita $b$, l'istante del picco della curva di potenza $p$, e l'istante della morte, $d$. L'autore ha precedentemente pubblicato diversi articoli riguardanti la sua teoria Evo-SETI (Evo-SETI significa Evoluzione e SETI) che riguarda l'evoluzione biologica sulla Terra negli ultimi 3.5 miliardi di anni, descritta come un incremento del numero di specie viventi dalla prima forma vivente (RNA, la prima molecola capace di riprodursi) fino al numero attuale di (diciamo) 50 milioni di Specie viventi sulla Terra. Le passate estinzioni in massa rendono questa evoluzione un processo stocastico, il cui valore medio è una funzione esponenziale, chiamato Moto Browniano Geometrico (GBM). In quegli articoli, un ciclo di vita, piuttosto che una Logell, era una b-lognormale, cioè una lognormale che parte da un instante $b$ (nascita), arriva fino al flesso discendente (s) e poi scende linearmente verso l'istante della morte a partire dal suo punto di flesso discendente $s$. La nostra scoperta matematica del Teorema del Luogo-dei-Picchi ha dimostrato che l'esponenziale GBM è il luogo dell'insieme dei picchi delle $b$-lognormali. Siccome le $b$-lognormali sono densità di probabilità, l'area sotto ciascuna di esse vale 1 (condizione di normalizzazione) e quindi, andando da sinistra a destra lungo l'asse dei tempi, le $b$-lognormali diventano sempre più "piccate", e quindi durano meno nel tempo. Questo "livello di civilizzazione” è quello che I fisici chiamano ENTROPIA (di Shannon) dell'informazione, cioè le Specie più evolute hanno un contenuto di informazione maggiore rispetto alle Specie meno evolute. Lautore ha anche dimostrato matematicamente che, per tutti gli esponenziali GBM, l'Entropia di Shannon delle $b$-lognormali cresce LINEARMENTE nel tempo. L'Orologio Molecolare, ben noto ai genetisti sin dal 1962, mostra che il numero di sostituzioni di basi nel DNA avvengono LINEARMENTE nel tempo poiché esse 
sono neutrali rispetto alla selezione darwiniana. La conclusione è che l'Orologio Molecolare e l'incremento LINEARE della EvoEntropy nel tempo sono proprio la stessa cosa! In alter parole, noi abbiamo derivato matematicamente LA LEGGE l'Orologio Molecolare come parte della nostra teoria Evo-SETI. Infine, questa entropia che aumenta linearmente è proprio la nuova SCALA EvoSETI per misurare l'evoluzione della vita non solo sulla Terra, ma anche sugli Esopianeti (la SCALA è misurata in bit).

In conclusione, la nostra invenzione della curva di Potenza Logell, descritta in questo articolo, fornisce un nuovo strumento matematico per la nostra descrizione matematica Evo-SETI della vita, della storia e del SETI.

Parole chiave

evoluzione biologica; orologio molecolare; entropia; SETI.

\section{PARTE 1:}

\section{CURVE LOGELL E LE LORO EQUAZIONI DELLA STORIA}

\section{INTRODUZIONE ALLE CURVE LOGELL “CON CICLO DI VITA FINITO”}

L'idea di partenza è semplice: cerchiamo di rappresentare il ciclo di vita di ogni essere vivente con soli tre istanti nel tempo: nascita, picco, morte (BPD). Nessun altro punto in mezzo è necessario. Cioè, nessun altro "punto di senilità" $s$ è richiesto come quello che appare in tutte le b-lognormali che l'autore ah pubblicato nella sua teoria Evo-SETI fino al 2017. Infatti, è più facile e naturale descrivere il ciclo di vita di un individuo nei soli termini di nascita, picco e morte, piuttosto che in termini di nascita, senilità e morte, poiché è incerto stabilire quando incominci la senilità e, in pratica, è difficile definirlo per qualunque individuo o per qualunque civilizzazione.

Si guardi la Figura 1.

La prima parte, sulla sinistra, cioè prima del picco $p$, è una semplice b-lognormale: parte all'istante della nascita $b$, cresce fino all'istante dell'adolescenza $a$ (punto di flesso ascendente della b-lognormale) (in realtà, l'istante dell'adolescenza si dovrebbe chiamare, più propriamente, "istante della pubertà" poiché segna l'inizio della capacità riproduttiva per quel dato individuo) e, finalmente, raggiunge il picco all'istante $p$ (punto di massimo, cioè il punto in cui la derivata prima della b-lognormale vale zero). Tutto quanto riportato finora è semplice materiale riguardante la b-lognormale, come abbiamo pubblicato a partire circa dal 2012.

Ma ora giunge la novità, cioè la seconda parte, quella a destra: cioè un'ellisse con vertice esattamente all'istante del picco $p$. Si noti come questa definizione implichi automaticamente che la linea tangente al picco è orizzontale, quindi lo stesso sia per la b-lognormale che per l'ellisse. Si noti anche come, dopo il picco, la curva scenda velocemente fino a quando raggiunge l'asse dei tempi all'istante della morte $d$ con una tangente perfettamente verticale. Quindi, questa nuova definizione di istante di morte $d$ è diversa dalla vecchia definizione di $d$, ottenuta facendo uso solamente delle b-lognormali, come abbiamo pubblicato prima del 2017.

E questa è la nuova CURVA LOGELL (b-LOGnormale più ELLisse), LIMITATA NEL TEMPO (cioè il suo supporto temporale va dalla nascita alla morte). Introduciamo qui la curva Logell per la prima volta in questo articolo e la analizziamo con risultati sorprendenti. 
History of the ROMAN CIVILIZATION as a LOGELL finite curve

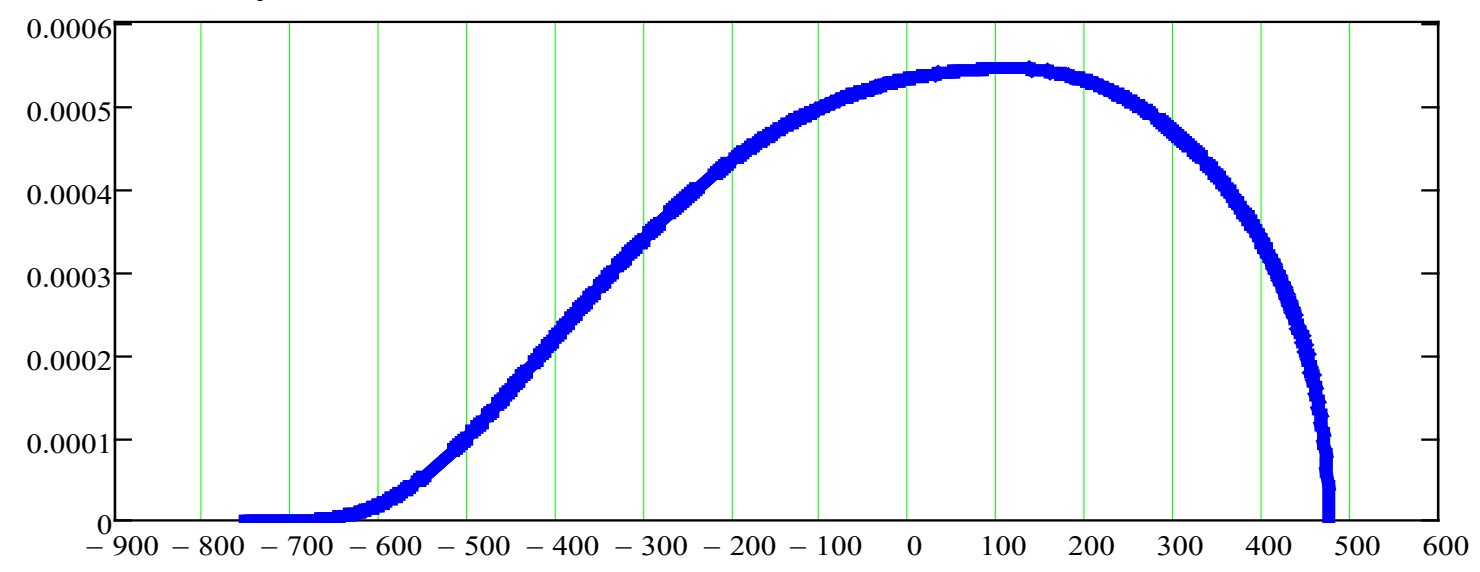

Figura 1. Rappresentazione della Storia della civilizzazione dell'Antica Roma come una curva LOGELL, limitata nel tempo. Roma fu fondata nel 753 A.C., cioè nell'anno -753, nella nostra notazione, o $b=-753$. Successivamente, la repubblica e l'impero romano (l'impero a partire dal primo imperatore, Augusto, all'incirca dopo il 27 A.C.) continuarono ad espandersi nei territori conquistati fino al picco (massima espansione, fino a Susa, nell'attuale Iran) avvenuta nell'anno 117 D.C., quindi $p=117$, sotto l'imperatore Traiano. Dopo, ha inizio il declino e la perdita di territori fino al collasso finale nel 476 D.C. $(d=476$, Romolo Augusto, l'ultimo imperatore). Quindi, sono necessari soltanto tre istanti nel tempo per riassumere tutta la Storia di Roma: $b=-753, p=117, d=476$. I numeri lungo l'asse verticale saranno spiegati nel seguito.

History of the ROMAN CIVILIZATION as a LOGPAR finite curve

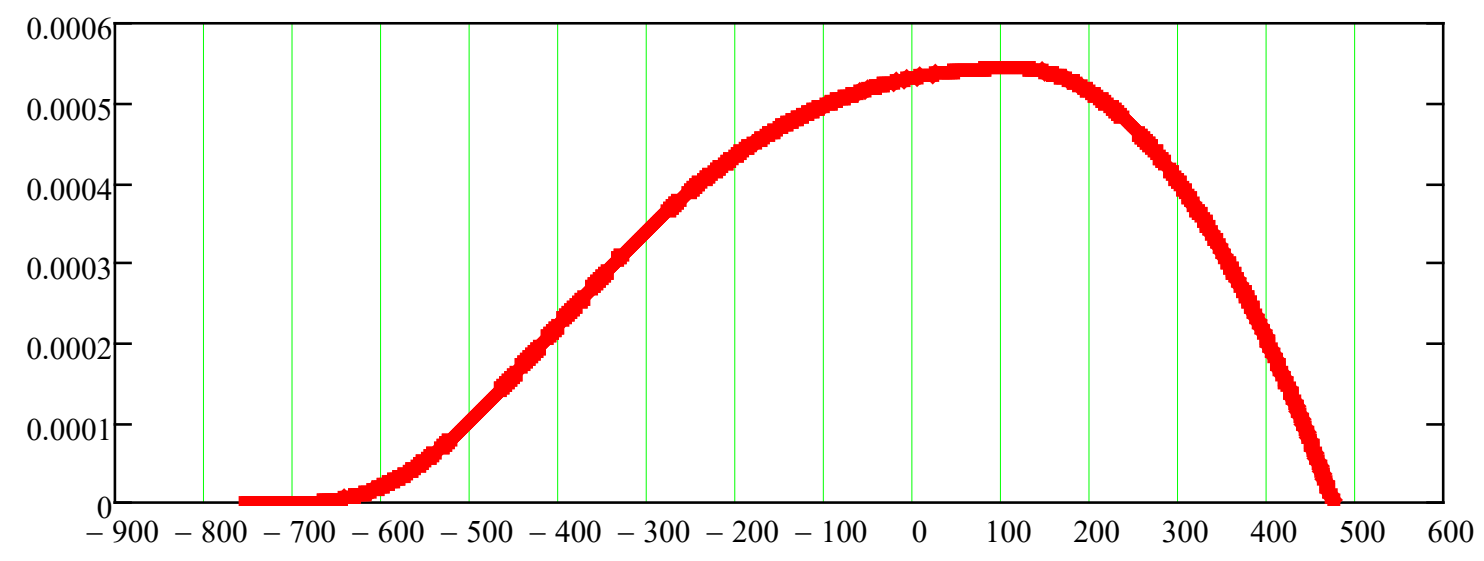

Figura 2. Rappresentazione della Storia della civiltà di Roma Antica come una curva limitata LOGPAR. Questa curva Logpar è composta, nel tempo, da una b-lognormale tra la nascita e il picco e da una parabola tra il picco e la morte. Fu usata dall'autore negli anni 2017 e 2018, nei rif. [1] and [2]. Si prega di leggere questi riferimenti per una trattazione matematica completa della Logpar, culminante nelle due Formule della Storia Logpar, che esprimono i due parametri della b-lognormale, $\mu$ (un numero reale) e $\sigma$ (un numero positivo) in termini di soltanto tre numeri reali $b, p$ e $d$, con la condizione $b<p<d$. 
Rome's LOGPAR (red) and LOGELL (red before peak, blue after peak)

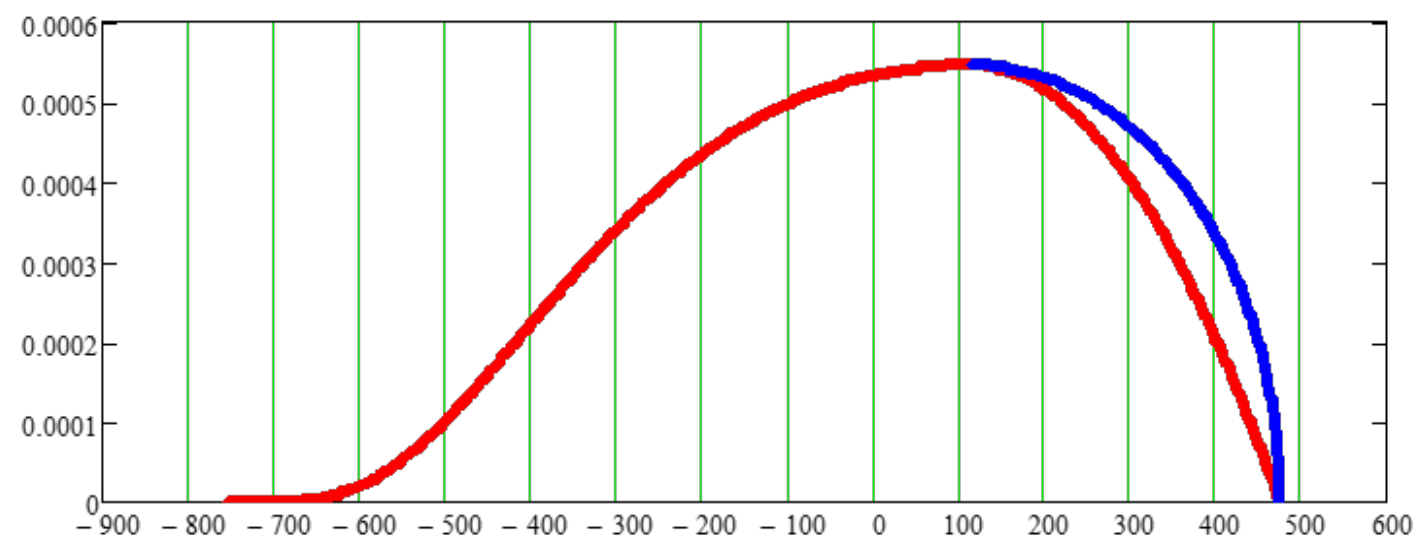

Figura 3. La differenza tra una LOGELL e una LOGPAR è solamente nel loro andamento tra il picco $p$ e la morte $d$ : un'ELLISSE per la LOGELL (in blu, qui sopra) e una PARABOLA per la LOGPAR (in rosso, qui sopra). La parte in comune della curva, prima del picco (in rosso, qui sopra) è una b-lognormale, cioè una funzione densità di probabilità lognormale ( $\mathrm{pdf}=$ probability density function) nel tempo, con inizio all'istante della nascita $b$ e picco all'istante $p$. In questo modo, il ciclo di vita limitato di ogni essere vivente o civiltà è una curva di POTENZA (Potenza misurata in Watt, come si fa in fisica) e l'area sotto questa curva di Potenza è l'ENERGIA totale di cui l'essere vivente o la civiltà hanno bisogno per la loro esistenza. Infatti, l'ENERGIA è proprio l'integrale della POTENZA nel tempo o, se si preferisce, la POTENZA è proprio la DERIVATA DELL'ENERGIA rispetto al tempo.

\section{EQUAZIONE DELL'ELLISSE NELLA PARTE DESTRA DELLA LOGELL}

Ora riporteremo, in un linguaggio opportunamente matematico, la descrizione data precedentemente della curva Logell.

Si consideri l'equazione di un'ellisse come nota a tutti gli studenti delle scuole superiori:

$$
\frac{x^{2}}{a^{2}}+\frac{y^{2}}{b^{2}}=1
$$

L'asse orizzontale è ora il tempo, con notazione $t$, e supporto $p<t<d$. Lungo l'asse verticale, abbiamo la variabile indipendente y che varia tra o e $P$. Si può quindi passare da (1) alla nuova equazione dell'ellisse grazie alle quattro sostituzioni

$$
\left\{\begin{array}{c}
x \rightarrow(t-p) \\
a \rightarrow(d-p) \\
y \rightarrow y \\
b \rightarrow P
\end{array}\right.
$$


Inserendo (2) in (1), quest'ultima diventa

$$
\frac{(t-p)^{2}}{(d-p)^{2}}+\frac{y^{2}}{P^{2}}=1 .
$$

Oppure

$$
\frac{y^{2}}{P^{2}}=1-\frac{(t-p)^{2}}{(d-p)^{2}}
$$

Risolvendo (4) per y e prendendo il segno più in fronte al radicale, quindi il ramo superiore dell'ellisse, si ottiene l'equazione desiderata del nostro quarto di ellisse in funzione del tempo $t$

$$
y(t)=P \sqrt{1-\frac{(t-p)^{2}}{(d-p)^{2}}} .
$$

A conferma, si può verificare che (5)immediatamente implica le due ovvie condizioni

$$
\left\{\begin{array}{l}
y(p)=P \\
y(d)=0
\end{array}\right.
$$

\section{EQUAZIONE DELLA b-LOGNORMALE NELLA PARTE SINISTRA DELLA LOGELL}

Per quanto riguarda la b-lognormale tra nascita e picco, cioè la parte sinistra della curva Logell, sono già noti tutti i dettagli matematici grazie ai molti articoli pubblicati precedentemente dall'autore, sull'argomento, ma verranno qui riassunte le equazioni principali, per completezza.

L'equazione della b-lognormale, con inizio in $b$, è

$$
\text { b_lognormal }(t ; \mu, \sigma, b)=\frac{e^{-\frac{(\log (t-b)-\mu)^{2}}{2 \sigma^{2}}}}{\sqrt{2 \pi} \sigma(t-b)} \text {. }
$$

con $\sigma>0$ e $-\infty<\mu<\infty$. L'autore ha fornito, nei rif. [1] e [2] le tavole con la lista delle principali equazioni che si possono dedurre da ( 7 ) e non saranno nuovamente dedotte qui. Ci limitiamo a ricordare che: 1) L'ascissa $p$ del picco di $(7)$ è data da

$$
p=b+e^{\mu-\sigma^{2}} \text {. }
$$

Dimostrazione. Si calcoli la derivata di (7) rispetto a $t$ e la si ponga uguale a zero. Poi, si risolva l'equazione risultante per $t$, che diventa ora $p$, e si trova così (8).

2) L'ordinata $P$ del picco di (7) è data da

$$
P=\frac{e^{\frac{\sigma^{2}}{2}-\mu}}{\sqrt{2 \pi} \sigma}
$$


Dimostrazione. Si scriva $p$ al posto di $t$ in (7) e si sostituisca(8)a $p$. Si semplifichi, quindi, per ottenere(9).

3) L'ascesa del puto di adolescenza $a$ (che si dovrebbe più propriamente chiamare "punto di pubertà") è l'ascissa del punto di flesso ascendente di (7). È data da

$$
a=b+e^{-\frac{\sigma \sqrt{\sigma^{2}+4}}{2}-\frac{3 \sigma^{2}}{2}+\mu}
$$

Dimostrazione. Si calcoli la derivata seconda di (7) rispetto a $t$ e la si ponga uguale a zero. Si risolva quindi l'equazione risultante per $t$, che diventa ora $a$, e si ottiene quindi (10) .

4) L'ordinata del punto di adolescenza è data da

$$
\frac{e^{-\frac{\sigma \sqrt{\sigma^{2}+4}}{4}+\frac{\sigma^{2}}{4}-\mu-\frac{1}{2}}}{\sqrt{2 \pi} \sigma}
$$

Dimostrazione. Si riscriva semplicemente $a$ al posto di $t$ in (7) e si inserisca (10) e si semplifichi il risultato.

Si noti ora che, nel contesto della teoria della Logell descritta in questo articolo, NON si può dire che (7) rispetti la condizione di normalizzazione della b-lognormale

$$
\int_{b}^{\infty} \mathrm{b} \_ \text {lognormal }(t ; \mu, \sigma, b) d t=1 .
$$

Infatti, (7) può solamente variare tra $b$ e $p$. Quindi, piuttosto che assumere (12), bisogna sostituire (12) con l'integrale di $(7)$ solamente tra $b$ e $p$. Fortunatamente, è possibile risolvere l'integrale in termini della funzione di errore, definite da

$$
\operatorname{erf}(x)=\frac{2}{\sqrt{\pi}} \int_{0}^{x} e^{-t^{2}} d t
$$

Infatti, l'integrale della b-lognormale (7) tra $b$ e $p$ risulta essere dato da

$$
\begin{aligned}
& \int_{b}^{p} \mathrm{~b}_{-} \operatorname{lognormal}(t ; \mu, \sigma, b) d t= \\
& =\int_{b}^{p} \frac{e^{-\frac{(\log (t-b)-\mu)^{2}}{2 \sigma^{2}}}}{\sqrt{2 \pi} \sigma(t-b)} d t=\frac{1+\operatorname{erf}\left(\frac{\sqrt{2} \ln (p-b)-\sqrt{2} \mu}{2 \sigma}\right)}{2}=
\end{aligned}
$$

Ora, inserendo (8) al posto di $p$ nell'argomento di erf, si ottiene una notevole semplificazione $\mu$ e $b$ scompaiono entrambi e rimane solamente $\sigma$. In aggiunta, la proprietà della funzione di errore $\operatorname{erf}(-x)$ $=-\operatorname{erf}(x)$ ci consente di riscrivere 


$$
=\frac{1+\operatorname{erf}\left(-\frac{\sigma}{\sqrt{2}}\right)}{2}=\frac{1-\operatorname{erf}\left(\frac{\sigma}{\sqrt{2}}\right)}{2} .
$$

In conclusione, l'area sotto la b-lognormale tra la nascita e il picco è data da

$$
\int_{b}^{p} \text { üüüüüüüü } \quad t \mu \sigma b d t=\frac{1-\operatorname{erf}\left(\frac{\sigma}{\sqrt{2}}\right)}{2}
$$

Questo risultato si dimostrerà essere di importanza chiave per ulteriori sviluppi descritti in questo articolo.

\section{AREA SOTTO L'ELLISSE NELLA PARTE DESTRA DELLA CURVA LOGELL TRA IL PICCO E LA MORTE}

Abbiamo già dimostrato che l'ellisse nella parte destra della curva Logell ha equazione . Ora vogliamo trovare l'area sotto l'ellisse tra il picco e la morte. Se ci si ricorda che l'area completa dell'ellisse con semiassi $a$ e $b$ è uguale a $\pi a b$, è ovvio che la nostra area è solo un quarto di questa. Lo stesso risultato si trova tramite la valutazione dell'integrale definito (si lascia questo calcolo al lettore, come esercizio)

$$
\int_{p}^{d} P \sqrt{1-\frac{(t-p)^{2}}{(d-p)^{2}}} d t=\frac{\pi}{4} P(d-p)
$$

\section{AREA SOTTO LA CURVA LOGELL COMPLETA TRA LA NASCITA E LA MORTE}

Siamo ora pronti a calcolare l'area completa $A$ sotto la curva Logell, che è data dalla somma delle equazioni (15) e (16), cioè

$$
\frac{1-\operatorname{erf}\left(\frac{\sigma}{\sqrt{2}}\right)}{2}+\frac{\pi}{4} P(d-p)=A
$$

Questa è una delle equazioni più importanti di questo articolo. Infatti, se volessimo che la Logell fosse una vera funzione densità di probabilità (pdf), dovremmo assumere in (17)

$$
A=1
$$

Ma, sorprendentemente, NON lo faremo!

Consideriamo, piuttosto, cosa stiamo facendo:

1) Stiamo creando un "modello matematico della Storia", dove l'"Evoluzione della Storia" di ogni Civilizzazione nel tempo è rappresentata da una curva Logell. 
2) In questo modello, si richiede soltanto la conoscenza di tre istanti nel tempo: $b, p$ e $d$.

3) L'area della curva completa dipende sia da $\sigma$ che da $\mu$, come si vede inserendo (9) al posto di $P$ in (17), cioè

$$
\frac{1-\operatorname{erf}\left(\frac{\sigma}{\sqrt{2}}\right)}{2}+\frac{e^{\frac{\sigma^{2}}{2}-\mu}}{\sqrt{2 \pi} \sigma} \cdot \frac{\pi}{4}(d-p)=A(\mu, \sigma) .
$$

4) Anche $p$ deve essere sostituito dalla sua espressione (8) in termini div $\sigma$ e $\mu$ per ottenere la nuova equazione

$$
\frac{1-\operatorname{erf}\left(\frac{\sigma}{\sqrt{2}}\right)}{2}+\frac{e^{\frac{\sigma^{2}}{2}}-\mu(\sigma)}{\sqrt{2 \pi} \sigma} \cdot \frac{\pi\left(d-b-e^{\mu(\sigma)-\sigma^{2}}\right)}{4}=A(\mu, \sigma) .
$$

5) La significa che (20) la nascita e la morte sono istanti fissati, ma la posizione del picco può muoversi liberamente tra di essi in accordo con i diversi esseri viventi o civilizzazioni che saranno considerati. In alter parole, (20) restituisce differenti valori numerici di $\sigma$ e $\mu(\sigma)$ a seconda di dove il picco si trova tra la nascita e la morte.

6) In aggiunta, vorremmo sbarazzarci della funzione di errore erf in (20). Come si può fare?

\section{L'AREA SOTTO LA CURVA LOGELL DIPENDE SOLO DA SIGMA. E ALLORA CI CALCOLIAMO LA DERIVATA DELL'AREA RISPETTO A SIGMA}

La risposta semplice all'ultima domanda 6) è "differenziando entrambi i membri di (20) rispetto a $\sigma$ ". Infatti, la derivata della funzione erf (13) è l'esponenziale "gaussiano"

$$
\frac{d \operatorname{erf}(x)}{d x}=\frac{2}{\sqrt{\pi}} \cdot e^{-x^{2}}
$$

e così, la funzione erf scomparirà differenziando (20) rispetto a $\sigma$. Infatti, la derivata del primo termine a sinistra del segno di uguale di è semplicemente, in accordo con (21),

$$
\frac{d}{d \sigma}\left[\frac{1-e r f\left(\frac{\sigma}{\sqrt{2}}\right)}{2}\right]=-\frac{e^{-\frac{\sigma^{2}}{2}}}{\sqrt{2 \pi}} .
$$

Per quanto riguarda la derivate rispetto a $\sigma$ del secondo termine a sinistra del segno di uguale di (20), prima di tutto notiamo che $\sigma$ compare tre volte nel termine stesso. Quindi la derivata è la somma di tre termini, ciascuno dei quali include la derivata di uno dei tre termini moltiplicato per gli altri due termini 
immutati. In equazioni, si ha:

$$
\begin{gathered}
\frac{d}{d \sigma}\left[\frac{1-e r f\left(\frac{\sigma}{\sqrt{2}}\right)}{2}+\frac{e^{\frac{\sigma^{2}}{2}-\mu}}{\sqrt{2 \pi} \sigma} \cdot \frac{\pi\left(d-b-e^{\mu-\sigma^{2}}\right)}{4}\right]= \\
=\frac{\sqrt{\pi} e^{-\frac{\sigma^{2}}{2}}}{2^{\frac{3}{2}}}-\frac{e^{-\frac{\sigma^{2}}{2}}}{\sqrt{2 \pi}}-\frac{\sqrt{\pi}\left(-e^{\mu-\sigma^{2}}+d-b\right) e^{\frac{\sigma^{2}}{2}-\mu}}{2^{\frac{5}{2}} \sigma^{2}}+ \\
-\frac{\sqrt{\pi}\left(e^{\mu-\sigma^{2}}-d+b\right) e^{\frac{\sigma^{2}}{2}-\mu}}{2^{\frac{5}{2}}} .
\end{gathered}
$$

Diverse forme alternative dell'equazione (23) sono possibili, e ciò è piuttosto disorientante. Però, con un manipolatore simbolico (l'autore si è servito del programma "Maxima" creato dalla NASA negli anni '6o per i difficili calcoli dei voli spaziali), alcuni ulteriori passi conducono alla seguente forma di (23):

$$
\begin{aligned}
& \frac{d A(\mu(\sigma), \sigma)}{d \sigma} \equiv \frac{d A(\sigma)}{d \sigma}= \\
& =\frac{e^{-\frac{\sigma^{2}}{2}+\mu} \sqrt{2}\left[\pi(d-b)\left(\sigma^{2}-1\right) e^{\sigma^{2}}+e^{\mu}\left(\sigma^{2}(\pi-4)+\pi\right)\right]}{8 \sqrt{\pi} \sigma^{2}} .
\end{aligned}
$$

Possiamo ancora semplificare inserendo (8) e facendo così scomparire $\mu$. Il risultato è

$$
\begin{aligned}
& \frac{d A(\sigma)}{d \sigma}= \\
& ==\frac{e^{-\frac{\sigma^{2}}{2}+\mu} \sqrt{2}\left\{\pi(d-p)\left(\sigma^{2}-1\right) e^{\sigma^{2}}+e^{\sigma^{2}}(p-b)\left[\sigma^{2}(\pi-4)+\pi\right]\right\}}{8 \sqrt{\pi} \sigma^{2}}= \\
& =\frac{e^{-\frac{\sigma^{2}}{2}+\mu} \sqrt{2} e^{\sigma^{2}}\left\{\pi(d-p)\left(\sigma^{2}-1\right)+(p-b)\left[\sigma^{2}(\pi-4)+\pi\right]\right\}}{8 \sqrt{\pi} \sigma^{2}}= \\
& =\frac{e^{\frac{\sigma^{2}}{2}+\mu} \sqrt{2}\left\{\sigma^{2}[\pi(d-b)+(\pi-4)(p-b)]-(\pi(d-p))\right\}}{8 \sqrt{\pi} \sigma^{2}} .
\end{aligned}
$$

La (25) è la derivata dell'area della Logell rispetto a sigma.

\section{7. “EQUAZIONI DELLA STORIA” ESATTE PER OGNI CURVA LOGELL}

Facciamo ora un ulteriore, cruciale passo nell'analisi della curva Logell: IMPONIAMO che la derivata dell'area rispetto a sigma, cioè , (25) sia zero

$$
\frac{d A(\sigma)}{d \sigma}=0
$$


Cosa significa?

La (26) è l'equivalente Evo-SETI di quello che è il PRINCIPIO DI MINIMA AZIONE nella fisica! Questa conclusione non si manifesta ora, ma lo farà al termine dell'articolo.

Per ora, riscriviamo la condizione imposta (26) grazie all'ultima espressione in (25) che, rimuovendo sia l'esponenziale, sia il denominatore, diventa

$$
\sigma^{2}[\pi(d-b)+(\pi-4)(p-b)]-(\pi(d-p))=0 .
$$

L'ultima equazione è una equazione quadratica in $\sigma$

$$
\sigma^{2}[\pi(d-b)+(\pi-4)(p-b)]=\pi(d-p)
$$

Risolta per $s^{2}$, diventa

$$
\sigma^{2}=\frac{\pi(d-p)}{\pi(d-b)+(\pi-4)(p-b)}
$$

Questo è il nuovo più importante risultato in questo articolo: la L'EQUAZIONE DELLA STORIA LOGELL PER $\sigma$

$$
\sigma=\frac{\sqrt{\pi} \sqrt{d-p}}{\sqrt{\pi(d-b)+(\pi-4)(p-b)}} .
$$

A parole, data la tripletta di ingresso $(b, p, d)$, la (30) restituisce immediatamente la $\sigma$ esatta della parte ellittica sinistra della curva Logell. Essa fu scoperta dall'autore il 4 settembre 2018, e portò non solo a questo articolo, ma all'introduzione dell'ENERGIA spesa nel ciclo di una vita da una creatura vivente o da una completa civilizzazione il cui andamento "potenza-nel-tempo" è dato dalla curva Logell, come si comprenderà meglio nel seguito.

Per il momento, ci limitiamo a calcolare il limite di entrambi i membri di (30) per $d \rightarrow \infty$, con il risultato

$$
\begin{aligned}
\lim _{d \rightarrow \infty} \sigma & =\lim _{d \rightarrow \infty} \frac{\sqrt{\pi} \sqrt{d-p}}{\sqrt{\pi(d-b)+(\pi-4)(p-b)}}= \\
& =\lim _{d \rightarrow \infty} \frac{\sqrt{\pi} \sqrt{d}}{\sqrt{\pi d}}=1 .
\end{aligned}
$$

Poiché già sappiamo che $s$ deve essere positiva, (31) dimostra che $\sigma$ può variare tra zero e uno solamente

$$
0<\sigma<1 .
$$

Si osservi che i due valori limite $\sigma \rightarrow 0$ e $\sigma \rightarrow 1$ non sono "fisici", perché non si può morire alla nascita oppure ad una età infinita, rispettivamente.

Similmente a (30) si ha, ovviamente, una EQUAZIONE LOGELL simile anche per $\mu$, che si deriva im- 
mediatamente da (8) e (30). A questo scopo, si consideri il logaritmo di (8)

$$
\mu=\ln (p-b)+\sigma^{2}
$$

e, con l'aiuto di , si trova la desiderata equazione Logell per $\mu$

$$
\mu=\ln (p-b)+\frac{\pi(d-p)}{\pi(d-b)+(\pi-4)(p-b)} .
$$

In conclusione, le nostre EQUAZIONI LOGELL CHIAVE DELLA STORIA sono

$$
\left\{\begin{array}{l}
\sigma=\frac{\sqrt{\pi} \sqrt{d-b}}{\sqrt{\pi(d-b)+(\pi-4)(p-b)}} \\
\mu=\ln (p-b)+\frac{\pi(d-p)}{\pi(d-b)+(\pi-4)(p-b)} .
\end{array}\right.
$$

\section{CONOSIDERAZIONI SULLE EQUAZIONI LOGELL DELLA STORIA}

Faciamo ora alcune considerazioni sulle Formule Logell (35) della Storia :

1) Le formule sono esatte, cioè non sono stati usati sviluppi in serie di Taylor.

2) Sono state derivate eguagliando a zero la derivata rispetto a $\sigma$ dell'area totale della curva Logell in .

3) Quindi, le Formule Logell della Storia sono le equazioni di un minimo (proveremo, nel seguito, che si tratta proprio di un minimo e non di un massimo) della funzione $A(\sigma)$ che esprime l'area totale (2o) come funzione di $\sigma$. Questo minimo è il PRINCIPIO di MINIMA ENERGIA della nostra Teoria Evo-SETI, cioè "nel nostro ciclo di vita, noi agiamo sempre in modo da minimizzare l'energia che stiamo usando". Un risultato tutt'altro che ovvio!

\section{COORDINATE DEL PICCO DELLA LOGELL IN TERMINI DEI SOLI $(b, p, d)$}

Di particolare importanza per tutte le future applicazioni della Logell sono le coordinate del picco $(p, P)$ espresso in termini della sola tripletta di ingresso $(b, p, d)$. Poiché l'ascissa $p$ del picco si considera nota, si deve solo derivare la formula dell'ordinata del picco $P$. Questa è prontamente ottenuta inserendo la Formula della Storia Logell (35) nell'espressione dell'altezza del picco (9). Dopo alcuni passaggi, si trova

$$
P=\frac{\sqrt{(\pi-4)(p-b)+\pi(d-b)}}{\sqrt{2} \pi \sqrt{d-p}(p-b)} e^{-\frac{\pi(d-p)}{2[(\pi-4)(p-b)+\pi(d-b)]}}
$$

\section{STORIA DI ROMA COME ESEMPIO DELL'UTILIZZO DELLE FORMULE DELLA STORIA DEL-}

\section{LA LOGELL}

Ritorniamo alla Storia di Roma come riassunta nella didascalia della Figura 1. 
Prima di tutto, scriviamo con cura i tre valori chiave numerici di ingresso nella Storia di Roma, che sono già stati menzionati nella didascalia della Figura 1:

$$
\text { Rome_input_triplet }=\left\{\begin{array}{l}
b=-753 \\
p=117 \\
d=476 .
\end{array}\right.
$$

Quindi, le Equazioni della Storia Logell (35) restituiscono immediatamente $\boldsymbol{i}$ valori numerici $\sigma$ e $\mu$ della Logell per Roma, che denoteremo da qui in poi $\sigma R$ e $\mu R$, rispettivamente

$$
\text { Rome_logell_doublet }\left\{\begin{array}{l}
\sigma R=0.602 \\
\mu R=7.131 .
\end{array}\right.
$$

Si giunge, ora, allo studio del picco della Logell. Sappiamo già, dalla (37), che l'ascissa del picco della civilizzazione di Roma fu nel 117 D.C. sotto Traiano

$$
p_{\text {Rome }}=117 \text {. }
$$

Ma il picco dell'ordinata per Roma si deve trovare grazie a (36). Si ottiene quindi

$$
P_{\text {Logell_Rome }}=6.358 \cdot 10^{-4}=0.0006358 \text {. }
$$

PARTE 2:

\section{ENERGIA COME AREA SOTTO LA CURVA DI POTENZA LOGELL}

\section{AREA SOTTO OGNI CURVA DI POTENZA LOGELL E IL SUO SIGNIFICATO COME “ENERGIA DEL CICLO DI VITA” DELL'ESSERE VIVENTE}

Le formule della storia Logell

$$
\left\{\begin{array}{l}
\sigma=\frac{\sqrt{\pi} \sqrt{d-p}}{\sqrt{\pi(d-b)+(\pi-4)(p-b)}} \\
\mu=\ln (p-b)+\frac{\pi(d-p)}{\pi(d-b)+(\pi-4)(p-b)} .
\end{array}\right.
$$

sono "molto simili" alle formule della storia Logpar (39) del rif. [1], cioè

$$
\left\{\begin{array}{l}
\sigma=\frac{\sqrt{2} \sqrt{d-p}}{\sqrt{2 d-(b+p)}} \\
\mu=\ln (p-b)+\frac{2(d-p)}{2 d-(b+p)} .
\end{array}\right.
$$

Da questo punto nell'articolo in poi, possiamo procedere in accordo a quanto definito nel rif. [1]. 
Qual è il significato fisico dell'area (20)?

Se si considera la curva Logell come la curva della potenza (misurata in watt) della civilizzazione di Roma lungo il corso completo della sua storia, allora l'area sotto questa curva, cioè l'integrale della Logell tra la nascita e la morte, è l'energia totale (misurata in joule) consumata dalla civilizzazione nel suo completo ciclo di vita:

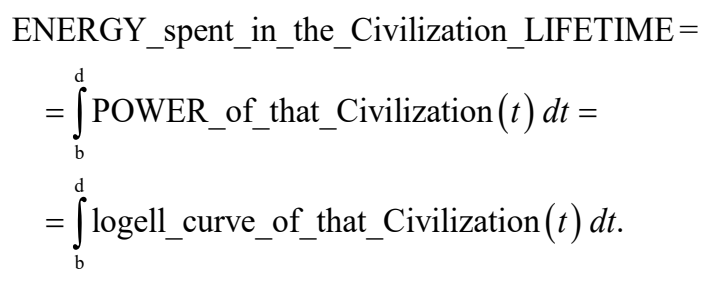

In altre parole, se conosciamo la curva di potenza di ogni essere vivente vissuto nel passato, quale una cellula, o un animale, o un essere umano, o una civilizzazione di esseri umani o qualunque altra forma vivente (inclusi gli Extraterrestri), l'integrale della curva di potenza, cioè della curva Logell, tra la nascita e la morte, è l'ENERGIA TOTALE consumata dal quella forma di vita durante tutto il ciclo della sua vita.

Un ulteriore punto riguarda l'ultima affermazione: se assumiamo che tutti gli umani abbiano, potenzialmente, la stessa quantità di energia da consumare durante tutto il loro ciclo di vita, allora la Logell di grandi uomini che "morirono da giovani" (come Mozart, per esempio) devono avere la stessa area sotto la loro Logell e quindi un picco molto più alto, poiché hanno vissuto per un periodo più breve degli altri uomini "comuni".

Consideriamo quindi la condizione che, usualmente, la morte arriva ovviamente dopo il picco ma, in casi eccezionali, la morte può arrivare proprio all'istante del picco (come, per esempio, per la morte in un incidente stradale), quindi

$$
d \geq p
$$

Ora vorremmo trovare il valore dell'ENERGIA AL PICCO del ciclo di vita di ciascuno. Perciò, prendendo il limite sinistro di per $d \rightarrow p$ e notando da che $\operatorname{erf}(0)=0$, troviamo

$$
\begin{aligned}
& \operatorname{Energy}(p)=\lim _{d \rightarrow p} \frac{1-\operatorname{erf}\left(\frac{\sigma}{\sqrt{2}}\right)}{2}= \\
& =\lim _{d \rightarrow p} \frac{1-\operatorname{erf}\left(\frac{\sqrt{\pi} \sqrt{d-p}}{\sqrt{2} \sqrt{\pi(d-b)+(\pi-4)(p-b)}}\right)}{2}= \\
& =\frac{1-\operatorname{erf}(0)}{2}=\frac{1}{2} .
\end{aligned}
$$

La può sembrare un risultato sorprendente: perché mai l'energia al picco, dovrebbe essere uguale proprio a $1 / 2$, e non a qualunque altro valore positivo (in joule)? No-problem: presto risolveremo anche l'estensione dell'Energia al picco, da $1 / 2$ a qualunque altro valore positivo come è già stato fatto nel rif. [1]. Ma, per il momento, rimaniamo soddisfatti di usare il valore convenzionale $1 / 2$ per semplificare i calcoli.

Al contrario, se consideriamo il limite di per $d \rightarrow \infty$ (questo limite è chiamato "limite dell'immortalità" poiché in questo caso l'essere vivente si suppone vivere per un tempo infinito) vediamo immediatamente che 


$$
\begin{aligned}
& \lim _{d \rightarrow \infty} A(\mu, \sigma)= \\
& =\lim _{d \rightarrow \infty}\left\{\frac{1-\operatorname{erf}\left(\frac{\sigma}{\sqrt{2}}\right)}{2}+\frac{e^{\frac{\sigma^{2}}{2}-\mu}}{\sqrt{2 \pi} \sigma} \cdot \frac{\pi}{4}(d-p)\right\}=\infty .
\end{aligned}
$$

In altre parole: "se vuoi vivere per un tempo infinito, hai bisogno di una quantità infinita di energia" (come è ovvio!).

Domanda successiva: qual è l'istante di tempo del minimo di energia? Saltiamo tutti lunghi calcli fatti con Maxima e scriviamo semplicemente i risultati:

$$
\begin{aligned}
& d_{\text {time_of_Logell_minimum_Energy }}= \\
& =b+\frac{\left(\sqrt{-\pi^{2}+8 \pi-12}+2\right)(p-b)}{\pi} .
\end{aligned}
$$

Questa è l'ascissa del minimo dell'Energia: potremmo dimostrare che si tratta veramente di un minimo, piuttosto che un massimo, calcolando la derivata seconda, ma non lo faremo per brevità.

Sottolineiamo piuttosto che, per il caso (37) dell'Antica Roma, (47) restituisce l'anno di minima energia di Roma nel 301 D.C. Questo era il tempo dell'imperatore Diocleziano (284 -305), sicuramente un periodo storico molto tormentato, poiché era il periodo dell'ultima e più grande persecuzione imperiale della Roma pagana contro i Cristiani. Nell'odierna Roma, i turisti che visitano la Basilica di "Santa Maria degli Angeli e dei Martiri", dovrebbero appunto pensare ai 4omila schiavi cristiani che morirono di fatica per costruire le Terme di Diocleziano, sito https:/it.wikipedia.org/wiki/Terme di Diocleziano aperte nel 306, cioè appunto nel tempo del minimo di civilizzazione della Roma pagana, dato dalla equazione (47) con la tripletta (37) della storia di Roma Antica.

Questo ovviamente significa che il nostro modello matematico della Storia si dimostra matematicamente CORRETTO !

\section{SCOPERTA DI UN ASINTOTO OBLIQUO DELLA FUNZIONE ENERGIA Energia(D) CON LA CRESCITA INDEFINITA DELL'ISTANTE DELLA MORTE $D$}

L'autore ha scoperto (il 4 settembre 2018) che l'energia totale della Logell (48) ha un asintoto obliquo per $d \rightarrow \infty$.

Prima di dedurre l'equazione dell'asintoto obliquo, comunque, è necessaria qualche attenta considerazione di cosa $d$ significhi. Abbiamo sempre detto che la teoria Logell descritta in questo articolo ha bisogno di tre quantità in ingresso $(b, p, d)$. Comunque, in questa sezione, considereremo valori sempre più grandi dell'istante della morte $d$ così ché l'area sotto la Logell, cioè l'energia dei fenomeni di cui la Logell è la potenza, possa assumere qualunque valor "grande". Così, in questa sezione, l'istante della morte $d$ diventa una sorta di nuova variabile indipendente $D$ piuttosto che una delle tre quantità in ingresso $(b, p, d)$. In altre parole, sarà fatta distinzione, con cura, tra

1) L'istante fisso e noto della morte $d$ e

2) La variabile indipendente, non fissa, $D$, che permetterà di estrapolare, nel futuro, la Logell con tre valori di ingresso fissi $(b, p, d)$.

Detto questo, l'ENERGIA totale fornita da Maxima inserendo (41) in (20) e risistemando, deve essere più 


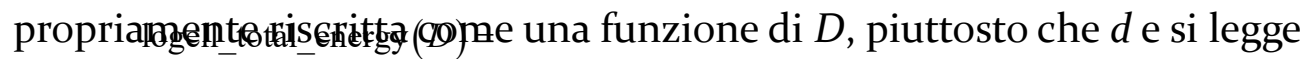

$$
\begin{aligned}
& =\frac{1-\operatorname{erf}\left(\frac{\sqrt{\pi} \sqrt{D-p}}{\sqrt{2} \sqrt{\pi(D-b)+(\pi-4)(p-b)}}\right)}{2}+ \\
& +\frac{\sqrt{D-p} \sqrt{\pi(D-b)+(\pi-4)(p-b)} e^{-\frac{\pi(D-p)}{2 \pi D+(2 \pi-8) p+(8-4 \pi) b}}}{2^{\frac{5}{2}}(p-b)} .
\end{aligned}
$$

La Figura 4 mostra sia l'energia totale della LOGELL in blu, sia l'energia totale della LOGPAR in rosso, quest'ultima descritta matematicamente in rif. [1].

Rome's LOGELL energy (blue) and LOGPAR energy (red) with asymptotes

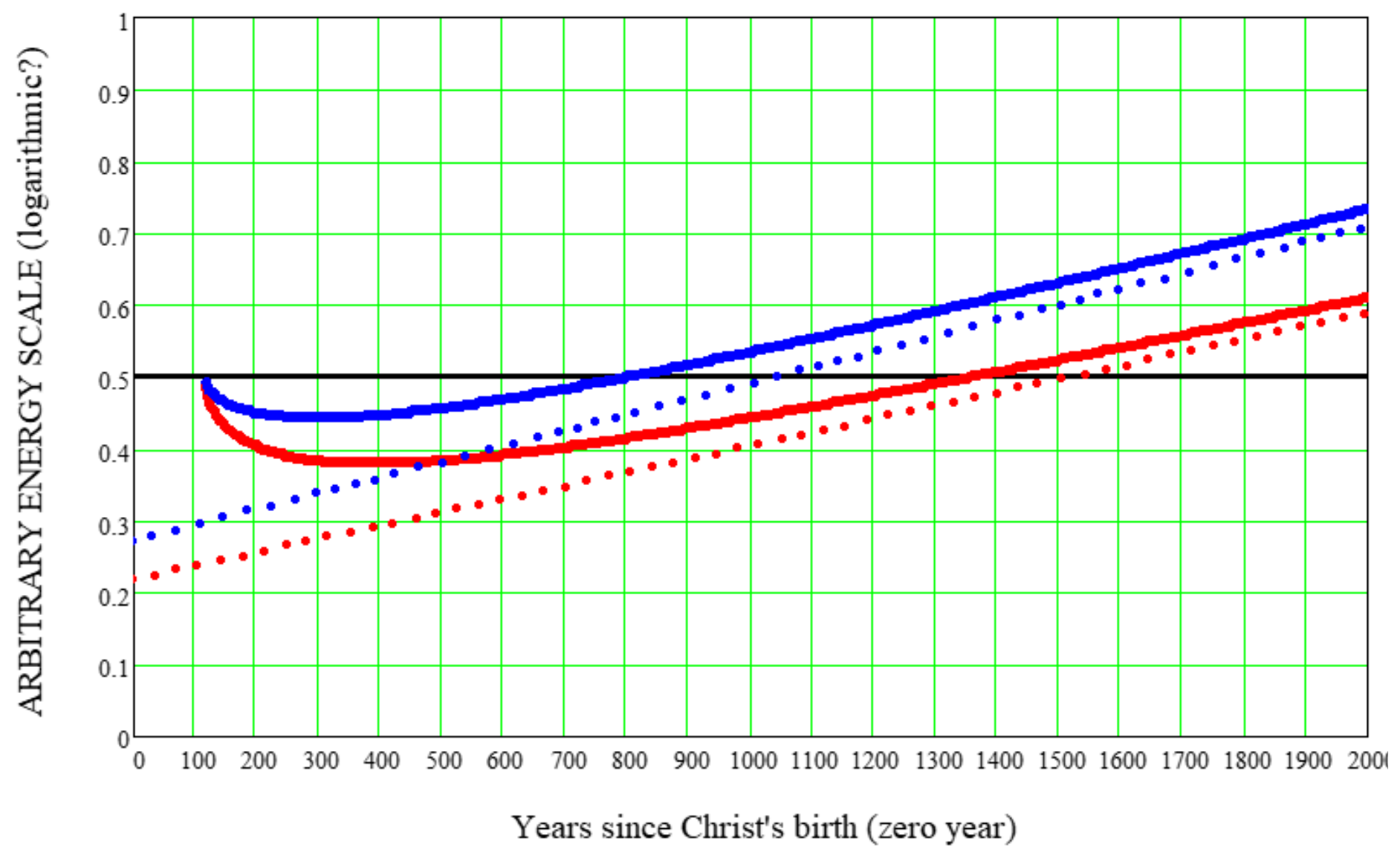

1) Figura 4. Energia totale di Roma come funzione di $D$ (cioè $d$, l'istante della morte considerata ora come la variabile indipendente): la curva continua in rosso e l'ENERGIA DELLA CURVA LOGPAR come descritta in rif. [1]. La linea retta a punti è il suo asintoto obliquo.

2) La curva continua in blu è l'ENERGIA DELLA CURVA LOGELL come nell'equazione (48) di questo articolo. La linea retta a punti in blu è il suo asintoto obliquo. 
Consideriamo, ora, la definizione di asintoto obliquo data nei libri di Analisi matematica: se il limite

$$
\lim _{D \rightarrow \infty}[\operatorname{Energy}(D)-(m D+q)]
$$

esiste, allora la curva di Energia Energy $(D)$ si avvicina sempre di più alla linea retta

$$
y_{\text {oblique_asymptote }}(D)=m D+q \text {. }
$$

Differenziando rispetto a $D$ vediamo immediatamente che il coefficiente angolare $m$ dell'asintoto obliquo è dato dal limite per $D \rightarrow \infty$ della derivate prima dell'energia (48).

Infatti, la derivata prima di è data dalla lunga espressione fornita da Maxima

$$
\begin{aligned}
& \frac{\mathrm{d}}{\mathrm{d} D} \text { logell_energy }=\left(\left(\pi^{2} D^{2}-4 \pi p D-2 \pi^{2} b D+4 \pi b D+\pi^{2} p^{2}-8 \pi p^{2}+16 p^{2}-2 \pi^{2} b p+20 \pi b p-32 b p+2\right.\right. \\
& \left.\left.\pi^{2} b^{2}-12 \pi b^{2}+16 b^{2}\right) \% \mathrm{e}^{2 \pi D+2 \pi p-8 p-4 \pi b+8 b}-\frac{\pi D}{2 \pi D+2 \pi p-8 p-4 \pi b+8 b}\right) /\left(2^{5 / 2}(p-b) \sqrt{D-p}\right. \\
& \left.(\pi D+(\pi-4) p+(4-2 \pi) b)^{3 / 2}\right)
\end{aligned}
$$

Prendendo il limite di per $D \rightarrow \infty$ otteniamo il coefficiente angolare $m$ dell'asintoto dell'energia:

$$
m=\lim _{D \rightarrow \infty} \frac{d \operatorname{Energy}(D)}{d D}=\frac{\sqrt{\pi}}{2^{\frac{5}{2}} \sqrt{e}(p-b)}=\frac{0.1900}{p-b} .
$$

Avremmo ottenuto lo stesso, ovviamente, se avessimo considerate il limite

$$
\lim _{D \rightarrow \infty} \frac{\operatorname{Energy}(D)}{D}=\lim _{D \rightarrow \infty} \frac{m D+q}{D}=m .
$$

Per quanto riguarda l'intersezione dell'asintoto con l'asse verticale, $q$, mostra che è data dal limite

$$
q=\lim _{D \rightarrow \infty}[\operatorname{Energy}(D)-m D] .
$$

Così, (50), (52) e Maxima portano al risultato

$$
q=\frac{1-\operatorname{erf}\left(\frac{1}{\sqrt{2}}\right)}{2}-\frac{\sqrt{e} \sqrt{\pi} p}{2^{\frac{5}{2}} \sqrt{e}(p-b)}+\frac{\sqrt{2} \pi-2^{\frac{3}{2}}}{4 \sqrt{e} \sqrt{\pi}} .
$$

In conclusione, l'equazione dell'asintoto obliquo all'Energia Logell (48) è data da 


$$
y(D)=\frac{\pi D+\pi(p-2 b)-4(p-b)}{2^{\frac{5}{2}} \sqrt{e} \sqrt{\pi}(p-b)}+\frac{1-e r f\left(\frac{1}{\sqrt{2}}\right)}{2}
$$

PARTE 3:

\section{POTENZA MEDIA NEL CICLO DI VITA DI UNA LOGELL}

\section{POTENZA MEDIA NEL CICLO DI VITA DI UNA LOGELL}

In questa sezione, considereremo la nozione di valore medio di una curva di potenza Logell.

Siccome abbiamo abbandonato la condizione di normalizzazione delle curve Logell, chiaramente non possiamo usare la stessa definizione di valore medio di una variabile casuale tipica della teoria della probabilità. È comunque semplice servirsi del Teorema del Valore Medio per gli Integrali, invece. Esso è una variazione del teorema del valore medio, che assicura che una funzione continua ha almeno un punto dove la funzione uguaglia la media della funzione.

Per tradurre il Teorema del Valor Medio per gli Integrali in una equazione matematica valida per le curve Logell, dobbiamo partire dall'equazione dell'Area, cioè l'equazione dell'energia della Logell (48) con $d$ sostituita da $\mathrm{D}$, e dividere quell'area per la lunghezza del segmento $(D-d)$, per ottenere il punto sull'asse verticale tale che l'area del rettangolo eguaglia l'Area. Questo è il Valore di Potenza Media in un ciclo di vita ed è dato da

$$
\begin{aligned}
& \text { Mean_POWER_over_a lifetime }=\frac{A(b, p, D)}{D-b}= \\
& =\frac{\operatorname{logel} \_ \text {total_energy }(D)}{D-b}= \\
& =\frac{1}{D-b} \cdot\left[\begin{array}{l}
\frac{1-\operatorname{erf}\left(\frac{\sqrt{\pi} \sqrt{D-p}}{\sqrt{2} \sqrt{\pi(D-b)+(\pi-4)(p-b)}}\right)}{2}+ \\
+\frac{\sqrt{D-p} \sqrt{\pi(D-b)+(\pi-4)(p-b)} e^{-\frac{\pi(D-p)}{2 \pi D+(2 \pi-8) p+(8-4 \pi) b}}}{2^{\frac{5}{2}}(p-b)}
\end{array}\right] .
\end{aligned}
$$

Solo per verificare la correttezza di , si consideri il limite della POTENZA Media in un ciclo di vita per $D \rightarrow \infty$. Il calcolo richiede l'utilizzo della regola di L'Hospital, e il risultato è

Asymptotic_Mean_Power_over_a_lifetime =

$$
\begin{aligned}
& =\lim _{D \rightarrow \infty}(\text { Mean_Power_over_a_lifetime })= \\
& =\lim _{D \rightarrow \infty}\left(\frac{\text { logell_total_energy }(D)}{D-b}\right)= \\
& =\frac{\sqrt{\pi}}{2^{\frac{5}{2}} \sqrt{e}(p-b)} .
\end{aligned}
$$


Ma, questo è, ovviamente, uguale al coefficiente angolare (52) dell'asintoto dell'energia della Logell, dal momento che il coefficiente angolare è proprio il limite della derivata dell'energia totale della Logell per $D \rightarrow \infty$. Quindi, il tutto ha senso.

Con questo, abbiamo completato lo studio della media sull'asse verticale, cioè l'asse della potenza. Comunque, si potrebbe ancora voler trovare, in un certo senso, "il valore medio di ciò che giace sull'asse orizzontale”, cioè il valor medio del ciclo di vita. Questo verrà fatto nella prossima sessione.

\section{PART 4:}

\section{VALOR MEDIO Di UN CICLO DI VITA PER UNA LOGELL}

\section{VALOR MEDIO DI UN CICLO DI VITA}

È naturale ricercare una espressione matematica che riporti il valor medio di un ciclo di vita, cioè il valore medio lungo l'asse dei tempi di tutto il segmento $(D-b)$ che rappresenta l'esistenza di un organismo vivente, o di una civilizzazione umana o addirittura di una civiltà extraterrestre.

Noi proponiamo la seguente definizione del valor medio di un ciclo di vita per una Logell:

$$
\begin{gathered}
\text { lifetime_mean_value }= \\
=\int_{b}^{p} t \cdot b \_ \text {lognormal }(t ; \mu, \sigma, b) d t+\int_{p}^{D} t \cdot \operatorname{ellipse}(t) d t=
\end{gathered}
$$

Inserendo la b-lognormale (7) e l'ellisse (5) in (59) quest'ultima diventa

$$
=\int_{b}^{p} t \cdot \frac{e^{-\frac{(\log (t-b)-\mu)^{2}}{2 \sigma^{2}}}}{\sqrt{2 \pi} \sigma(t-b)} d t+\int_{p}^{D} t \cdot P \sqrt{1-\frac{(t-p)^{2}}{(D-p)^{2}}} d t .
$$

Il primo integrale si può risolvere in termini di funzione d'errore $\operatorname{erf}(x)$ data da (13), e il risultato è

$$
\begin{aligned}
& \int_{b}^{p} t \cdot \frac{e^{-\frac{(\log (t-b)-\mu)^{2}}{2 \sigma^{2}}}}{\sqrt{2 \pi} \sigma(t-b)} d t= \\
&= \frac{e^{\frac{\sigma^{2}}{2}+\mu}\left[1-\operatorname{erf}\left(\frac{\sigma^{2}-\log (p-b)+\mu}{\sqrt{2} \sigma}\right)\right]}{2}+ \\
&+\frac{b\left[1-\operatorname{erf}\left(\frac{\log (p-b)-\mu}{\sqrt{2} \sigma}\right)\right]}{2}=
\end{aligned}
$$

Che può essere ulteriormente semplificato grazie a (8), con il risultato 


$$
\begin{aligned}
\int_{b}^{p} t & \cdot \frac{e^{-\frac{(\log (t-b)-\mu)^{2}}{2 \sigma^{2}}}}{\sqrt{2 \pi} \sigma(t-b)} d t= \\
\quad & =\frac{e^{\frac{\sigma^{2}}{2}+\mu}[1-\operatorname{erf}(\sqrt{2} \sigma)]}{2}+\frac{b\left[1-\operatorname{erf}\left(\frac{\sigma}{\sqrt{2}}\right)\right]}{2} .
\end{aligned}
$$

Riscrivendo ora (62) in termini delle Formule della Storia Logell (35), il primo dei due integrali (6o) prende la forma lunga ma esatta data da Maxima, come segue:

$$
\begin{aligned}
& \int_{b}^{p} t \cdot \frac{e^{-\frac{(\log (t-b)-\mu)^{2}}{2 \sigma^{2}}}}{\sqrt{2 \pi} \sigma(t-b)} d t= \\
& -\frac{(p-b) \% \mathrm{e}^{\frac{3 \pi(d-p)}{2((\pi-4)(p-b)+\pi(d-b))}} \operatorname{erf}\left(\frac{\sqrt{2} \sqrt{\pi} \sqrt{d-p}}{\sqrt{(\pi-4)(p-b)+\pi(d-b)}}\right)}{2}-\frac{b \operatorname{erf}\left(\frac{\sqrt{\pi} \sqrt{d-p}}{\sqrt{2} \sqrt{(\pi-4)(p-b)+\pi(d-b)}}\right)}{2} \\
& \frac{(b-p) \% \mathrm{e}^{\frac{3 \pi(d-p)}{2((\pi-4)(p-b)+\pi(d-b))}}}{2}+\frac{b}{2}
\end{aligned}
$$

Per quanto riguarda il secondo integrale in (6o), cioè l'integrale dell'ellisse, è prontamente risolto come segue

$$
\int_{p}^{D} t \cdot P \cdot \sqrt{1-\frac{(t-p)^{2}}{(D-p)^{2}}} d t=
$$

$\left(\left(3 p^{3}-6 d p^{2}+3 d^{2} p\right) \operatorname{asin}\left(\frac{D-p}{p-d}\right)+2 p^{3}-6 d p^{2}+6 d^{2} p-2 d^{3}\right) P+\sqrt{-D+2 p-d} \cdot \sqrt{D-d}$ $\left.\left(2 D^{2}-p D-3 p^{2}+4 d p-2 d^{2}\right) P\right) /(6 p-6 d)$ 
Inserendo, al posto di $P$, la sua espressione (36), dopo alcuni passaggi, si conclude che l'integrale dell'ellisse è dato da

$$
\begin{aligned}
& \int_{p}^{D} t \cdot P \cdot \sqrt{1-\frac{(t-p)^{2}}{(D-p)^{2}}} d t= \\
& \left(\left(\sqrt{\pi(D-b)+(\pi-4)(p-b)} \% \mathrm{e}^{2(\pi(D-b)+(\pi-4)(p-b))}+\frac{\pi(p-D)}{\pi D+\pi p-4 p-2 \pi b+4 b}\right.\right. \\
& \left(\left(3 p^{3}-6 d p^{2}+3 d^{2} p\right) \operatorname{asin}\left(\frac{D-p}{p-d}\right)+2 p^{3}-6 d p^{2}+6 d^{2} p-2 d^{3}\right) /(\sqrt{2} \pi(p-b) \sqrt{D-p})+(\sqrt{-D+2 p-d} \cdot \sqrt{D-d} \\
& \left.\sqrt{\pi(D-b)+(\pi-4)(p-b)}\left(2 D^{2}-p D-3 p^{2}+4 d p-2 d^{2}\right) \% \mathrm{e}^{2(\pi(D-b)+(\pi-4)(p-b))}+\frac{\pi(p-D)}{\pi D+\pi p-4 p-2 \pi b+4 b}\right) /(\sqrt{2} \pi \\
& (p-b) \sqrt{D-p})) /(6 p-6 d)
\end{aligned}
$$

In conclusione, il ciclo di vita medio si trova dalla somma di (63) e (65) e diventa

lifetime_mean_value =

$$
\begin{aligned}
& -\frac{(p-b) \% \mathrm{e}^{\frac{3 \pi(d-p)}{2((\pi-4)(p-b)+\pi(d-b))}} \operatorname{erf}\left(\frac{\sqrt{2} \sqrt{\pi} \sqrt{d-p}}{\sqrt{(\pi-4)(p-b)+\pi(d-b)}}\right)}{2}-\frac{b \operatorname{erf}\left(\frac{\sqrt{\pi} \sqrt{d-p}}{\sqrt{2} \sqrt{(\pi-4)(p-b)+\pi(d-b)}}\right)}{2}- \\
& \frac{\frac{3 \pi(d-p)}{2(b-p) \% \mathrm{e}^{2((\pi-4)(p-b)+\pi(d-b))}}+\frac{b}{2}}{2}
\end{aligned}
$$

$\left(\left(\sqrt{\pi(D-b)+(\pi-4)(p-b)} \% \mathrm{e}^{\frac{\pi(D-p)}{2(\pi(D-b)+(\pi-4)(p-b))}}+\frac{\pi(p-D)}{\pi D+\pi p-4 p-2 \pi b+4 b}\right.\right.$

$\left.\left(\left(3 p^{3}-6 d p^{2}+3 d^{2} p\right) \operatorname{asin}\left(\frac{D-p}{p-d}\right)+2 p^{3}-6 d p^{2}+6 d^{2} p-2 d^{3}\right)\right) /(\sqrt{2} \pi(p-b) \sqrt{D-p})+(\sqrt{-D+2 p-d} \cdot \sqrt{D-d}$

$\left.\sqrt{\pi(D-b)+(\pi-4)(p-b)}\left(2 D^{2}-p D-3 p^{2}+4 d p-2 d^{2}\right) \% \mathrm{e}^{\frac{\pi(D-p)}{2(\pi(D-b)+(\pi-4)(p-b))}}+\frac{\pi(p-D)}{\pi D+\pi p-4 p-2 \pi b+4 b}\right) /(\sqrt{2} \pi$ $(p-b) \sqrt{D-p})) /(6 p-6 d)$ 
Per dare un esempio numerico, troviamo il ciclo di vita medio della civilizzazione di Roma. Il primo integrale , per mezzo della tripletta di ingresso di Roma (37), restituisce il valore numerico della b-lognormale media, cioè

$$
\text { mean_value_of_Rome_b-lognormal }=-35.6
$$

Questo significa quattro anni prima della battaglia di Azio https://en.wikipedia.org/wiki/Battle of Actium, combattuta il 2 settembre 31 A.C.: un evento cruciale che vide l'Egitto di Cleopatra assorbito come una provincia romana.

D’altra parte, l'integrale del quarto di ellisse (16) e la tripletta di ingresso di Roma (37) restituiscono, per la media del quarto di ellisse, l'anno

Rome_quarter-of-ellipse_mean_value $=49.1$.

Nell'anno 49 D.C. era imperatore Claudio (41-54 A.D.), e, fatto molto più importante, erano trascorsi appena 16 anni dalla crocifissione di Gesù Cristo a Gerusalemme. Così, sommando le due equazioni (67) e (68), raggiungiamo l'importante conclusione che il valore medio della curva di potenza LOGELL della storia completa di Roma, cade proprio attorno all'anno 13.5 D.C., cioè nel tempo di Augusto, il primo imperatore di Roma (egli morì il 19 agosto del 14 D.C.):

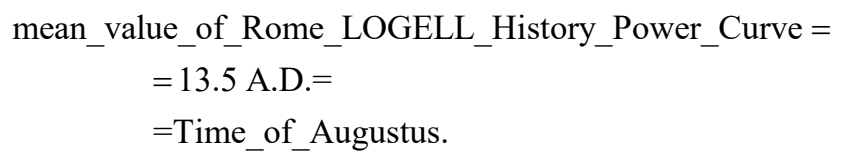

Questo è un risultato degno di nota. La nostra teoria Evo-SETI, nella forma della LOGELL descritta in questo articolo, predice che "l'anno più importante di Roma fu... nel tempo di Augusto, e proprio quando Gesù Cristo stava vivendo il suo periodo di transizione dalla giovinezza all'età adulta”. Il periodo di formazione di gran lunga più importante per la vita di ogni uomo, e ancora di più per la vita Gesù!

\section{PARTE 5:}

\section{CONCLUSIONI: QUALE E' MEGLIO? LA LOGELL O LA LOGPAR?}

\section{CONCLUSIONI A PROPOSITO DELLA CIVILTA' DI ROMA}

In questo articolo, abbiamo discusso per la prima volta due modelli matematici alternativi per la teoria Evo-SETI:

1) Il modello LOGELL, dove la vita è descritta da una curva di Potenza composta da una b-lognormale tra la nascita e il picco più un quarto di un'ellisse tra il picco e la morte.

2) Il modello LOGPAR, dove la vita è descritta da una curva di Potenza composta da una b-lognormale tra la nascita e il picco più una parabola discendente tra il picco e la morte.

Quale descrive la vera storia di una civilizzazione?

Per il caso di Roma, i due modelli forniscono i seguenti risultati:

1) LOGELL: minimo dell'energia al tempo di Diocleziano, cioè quasi al tempo della cruciale transizione 
tra la Roma pagana e quella cristiana (313 D.C. Editto di Milano di Constantino e Licinio). Secoli dopo questo punto di minimo si avrà la completa ripresa (cioè al passato livello di Traiano, livello del 117 D.C.) solo intorno all'anno 8oo D.C. cioè grazie a Carlo Magno.

2) LOGPAR: minimo dell'energia intorno all'anno 378 D.C. (anno della prima seria sconfitta inflitta dai barbari Visigoti ai Romani nella battaglia di Adrianopoli) e poi, secoli dopo, completa ripresa soltanto alla fine del Medioevo, cioè attorno al 1400 D.C. (Rinascimento italiano).

L'opinione personale dell'autore è che il modello LOGPAR sia più appropriato di quello LOGELL, almeno nel caso della storia di Roma.

\section{CONCLUSIONI SULLA TEORIA EVO-SETI FATTE NELL'ANNO 2019}

Gli astronomi scoprono sempre più esopianeti grazie ad osservazioni da Terra o grazie a missioni spaziali, come le missioni spaziali “Kepler”, “Gaia”, “Tess” e altre missioni spaziali future. Di conseguenza, una recente stima porta a 40 miliardi il numero di pianeti della misura della Terra orbitanti nelle zone abitabili di stelle simili al Sole e nane rosse nella Via Lattea. Con numeri così grandi di "Terre possibili" in vista, l'Astrobiologia e il SETI stanno diventando campi di ricerca sempre più attraenti per giovani scienziati.

Articoli matematicamente innovativi come quelli della Teoria Evo-SETI, che rivelano inaspettate relazioni come quella tra l'Orologio Molecolare e l'Entropia delle $b$-lognormali nella Teoria Evo-SETI, dovrebbero dunque essere ben accolti.

Ma nei rif. [1], [2] e in questo articolo, è stato fatto di più che nei precedenti articoli Evo-SETI.

Mentre si preservano tutti i vantaggi delle funzioni densità di probabilità b-lognormali, adesso queste sono state mantenute solo nella prima parte della curva: quella tra la nascita e il picco. La seconda parte, tra il picco e la morte, è stata sostituita, in questo articolo, per la prima volta, da un semplice quarto di ellisse discendente, evitando così ogni punto di flesso come il punto di "senilità" tipico delle b-lognormali, che era così difficile da stimare numericamente nella maggior parte dei casi.

Così, sia le curve LOGELL, che le curve LOGPAR, hanno molto semplificato la descrizione di ogni fenomeno limitato nel tempo come il ciclo di vita di una cellula, o di un umano, o di una civilizzazione (come quella di Roma, usata in questo articolo come esempio) o anche di una civiltà ET.

In altre parole, abbiamo abbandonato la condizione di normalizzazione delle b-lognormali, conservando solo la loro forma analitica, ma NON la loro area unitaria, cioè NON la condizione di normalizzazione. Questo ha trasformato entrambe le curve Logpar e Logell in curve di potenza, sia nel senso popolare dove "potenza" significa "potere politico \& militare", sia nel senso strettamente fisico, dove "potenza" significa una curva misurata in Watt.

E l'area sotto la Logpar e la Logell è proprio l'ENERGIA associate al ciclo di vita totale tra la nascita e la morte.

Così, per la prima volta dalla creazione della Teoria Evo-SETI, abbiamo aggiunto l'ENERGIA all'ENTROPIA già precedentemente considerata.

E l'energia e l'entropia sono i due pilastri della Termodinamica classica, rendendo l'Evo-SETI anche più semplicemente applicabile ad un amplissimo gruppo di fenomeni biologici e fisici.

Infine, c'è un ulteriore passo cruciale che noi abbiamo fatto introducendo le Logpar e le Logell. Senza averlo finora menzionato, abbiamo di fatto ottenuto qualcosa di simile al PRINCIPIO DI MINIMA AZIONE per la Teoria Evo-SETI.

Il futuro ci dirà se le nostre idee qui presentate sono corrette e largamente applicabili sia all'Astrobiologia che al SETI, e anche allo studio della "Storia in Matematica". 


\section{RIFERIMENTI}

Maccone, C. 2018. "Energy of Extraterrestrial Civilizations according to Evo-SETI Theory." Acta Astronautica 144, 202-213.

———. 2018. "Life Expectancy and Life Energy according to Evo-SETI Theory." International Journal of Astrobiology 18 (1) 1-11. 